Marcello Giusto

Università Adam Mickiewicz di Poznań

https://doi.org/10.18778/8220-506-0.06

\title{
CAROSELLO: SPECCHIO E STRUMENTO SOCIALE, CULTURALE E LINGUISTICO DELL'ITALIANITÀ
}

Riassunto: Sulla base di un campione esemplificativo, si intende svelare l'importante ruolo di Carosello, il contenitore televisivo di messaggi promozionali creato dalla RAI nel 1957, visto attraverso il prisma dell'italianità, non solo per la sua struttura innovativa, originale e integralmente italiana, ma anche per l'enorme influsso che ebbe sulla cultura e sulla società. Da un lato personaggi, testimonial, slogan, neologismi e frasi fatte reiterati entrarono nell'uso linguistico e nell'immaginario comune, dall'altro si fecero portatori del nuovo messaggio della società dei consumi, predisposto sulla base di un sistema di valori sviluppati intorno all'italianità ed espressi per mezzo di elementi retorici stilisticamente e persuasivamente funzionali.

Parole chiave: carosello, pubblicità televisiva, sociolinguistica italiana, pragmatica, retorica.

\begin{abstract}
Carosello: mirror and social, cultural and linguistic instrument of the Italian spirit. The aim of the article "Carosello: Italianness' mirror and social, cultural and linguistic device" is to reveal the important role of "Carosello", the television container of promotional messages created by RAI in 1957, seen through the key of Italianness. It was an entirely Italian invention, with an innovative and original structure, which also influenced culture and society. The analysis of selected samples attempts to demonstrate how his testimonials, repeated slogans, neologism and idioms entered the common language and imaginary especially as bearers of the new consumer society's message organised in a system of values developed around Italianness and expressed through stylistically and persuasively functional rhetorical elements.
\end{abstract}

Keywords: television advertisement, Italian sociolinguistics, pragmatics, rhetoric. 


\section{Introduzione}

In base alla definizione di italianità di Treccani ${ }^{1}$, essa va intesa come ciò che è considerabile distintamente italiano o degli italiani, sia esso la lingua, l'indole, il costume, la cultura o la civiltà. Tali aspetti si legano al senso e alla coscienza di appartenenza, nonché all'essere e sentirsi italiano. In questa sede, concentrandosi in particolare sui fattori esterni e sulle caratteristiche linguistiche intrinseche del fenomeno, si intende verificare in quale modo il complesso mondo di Carosello, filtrato attraverso il quadro descritto, si iscriva nella storia della pubblicità televisiva italiana come strumento che ha coadiuvato la formazione dell'identità degli italiani.

\section{La cornice storico-sociale}

Alla fine della Seconda Guerra mondiale, l'Italia era un Paese profondamente devastato, prettamente rurale e segnato dal problema dell'analfabetismo. Se nei decenni seguenti ebbe inizio la fase della ricostruzione economica, il primo vero impulso verso il cambiamento arriva con l'avvento della televisione. Dopo un periodo di programmazione sperimentale, la televisione italiana cominciò ad emettere ufficialmente e regolarmente, su un unico canale, da domenica 3 febbraio 1954. Da allora, la televisione entrò nelle case e nella vita degli italiani con un'immediata popolarità crescente: se nel 1954 gli apparecchi erano solo 90.000 , l'anno successivo si rilevano già sette milioni di telespettatori. Essendo un elettrodomestico inizialmente non alla portata di tutti, solo il 3,1 e il 12,5\% degli italiani guardava la tv rispettivamente da casa propria o da amici e parenti, mentre la maggioranza (il 45\%) da locali pubblici (Dorfles 1998: 9). La televisione rivoluzionò sin da subito le abitudini delle persone, incentivando un modello di socializzazione fino ad allora sconosciuto. Inizialmente, il palinsesto seguiva la scansione della vita quotidiana: non c'erano programmi durante le ore lavorative, le trasmissioni per i ragazzi occupavano sempre la fascia oraria del secondo pomeriggio, mentre il momento più atteso era quello serale con film, teatro, varietà, inchieste che avevano una collocazione fissa durante la settimana.

Sul modello dell'inglese BBC e coerente agli ideali democristiani del governo italiano dell'epoca, proprio il fine didattico e pedagogico è alla base delle linee editoriali della televisione firmata RAI degli albori, ovvero dell'era che denominata paleotelevisione. La tv deteneva il ruolo dell'educatore, del divulgatore di cultura anche attraverso le forme di spettacolo (adattamenti

1 www.treccani.it/vocabolario/italianita/ [26/02/2020]. 
delle grandi opere letterarie, prose, programmi culturali, quiz che fondono in sé intento didattico, leggerezza e linguaggio dal tono serio), e di scuola di lingua (Simone 1987; De Mauro 1993: 437-443), mirando alla crescita del livello d'informazione e cercando di far acquisire un patrimonio linguistico comune alla diversificata popolazione analfabeta italiana, che spesso conosceva solo il dialetto. La televisione rappresentò uno strumento di unificazione nazionale, sia dal punto di vista linguistico che socio-culturale. Si tratta di una prima traccia nella ricerca dell'italianità attraverso la formazione di un'identità italiana, eliminando le disuguaglianze culturali, sociali e regionali, consentendo allo spettatore di autoriconoscersi in quella nazione e possedere gli strumenti che gli permetteranno di meglio entrare a far parte dell'imminente era moderna (Simonelli e Colombo 2004: 14-15).

\section{Carosello}

In questa cornice nasce la pubblicità televisiva italiana. Rispetto al modello dello spot, già utilizzato all'estero, in Italia venne creato un programma apposito composto di soli messaggi pubblicitari, al fine di evitare l'interruzione delle trasmissioni come richiedeva la Convenzione stipulata fra RAI e Ministero delle Poste e delle Telecomunicazioni ${ }^{2}$, il cui art. 19 stabiliva come la pubblicità dovesse essere introdotta "nelle forme più convenienti per non recare pregiudizio alla bontà dei programmi”. Poiché la réclame di allora non godeva di una buona dignità era necessaria una forma a metà fra spettacolo e teatro che permettesse di "porgerla" allo spettatore in modo gradevole, riservando un piccolo spazio finale al comunicato pubblicitario, l'esca che coglie l'attenzione dello spettatore (Molinari 2019: 23). Dopo una serie di progetti pilota, nacque Carosello, programma trasmesso dal 3 febbraio 1957 al $1^{\circ}$ gennaio 1977, come appuntamento fisso alle 20:50, subito dopo il telegiornale della sera e prima delle trasmissioni serali.

Le rigide norme della RAI prevedevano, fra l'altro, che Carosello iniziasse e si chiudesse con una sigla, fosse costituito da quattro o cinque comunicati, intervallati da un siparietto, e aventi ognuno una precisa struttura. La prima parte era il cosiddetto pezzo, una scenetta d'apertura che non poteva essere trasmessa identica una seconda volta, della durata fra i 65 e i 115 secondi (a seconda dell'anno di riferimento), nella quale non poteva essere nominato il prodotto. Poteva essere girato dal vivo (con la partecipazione di attori

2 Approvata con Decreto del Presidente della Repubblica del 26 gennaio 1952, n. 180, consultabile all'indirizzo: www.edizionieuropee.it/LAW/HTML/47/ zn84_01_015.html [28/02/2020]. 
e comparse), in forma animata o in "passo uno" (con oggetti reali in movimento). Si caratterizzava per uno stile brillante e comico, e le scene si rifacevano al teatro della rivista, all'avanspettacolo e ai film, in particolare alla commedia, dunque a forme narrative che il pubblico conosceva bene; non mancavano comunque altri generi, soprattutto d'intrattenimento musicale o profondamente educativi. Seguiva poi il cosiddetto codino di chiusura, della durata di massimo 35 secondi (30 dal 1973), che costituiva la "vera" pubblicità, poiché il prodotto vi veniva realmente reclamizzato. Si caratterizzava dunque per un linguaggio più referenziale e tecnico. A collegare pezzo d'apertura e codino c'era sempre una frase d'aggancio studiata appositamente per fungere da chiave di volta che permettesse il quasi inaspettato passaggio dalla parte iniziale di intrattenimento al messaggio pubblicitario vero e proprio, con un certo effetto sorpresa.

Nella seconda metà degli Anni 70, nonostante un'audience sempre elevata, i vertici della Rai chiudono il programma. Un fattore imputabile è l'onerosità del limitato spazio pubblicitario (dal costo iniziale di 1.500.000 Lire si era giunti nel 1976 a quasi sette milioni) ${ }^{3}$ inaccessibile e poco conveniente per le aziende. Inoltre, il format venne additato come "troppo italiano": l'italianità a cui Carosello doveva il successo, strideva con la realtà degli Anni 70, con i mercati sempre più internazionalizzati che esigevano pubblicità veloci e adattabili a lingue e culture diverse. Carosello non possiede queste carte e, non essendo al passo con una società che vuole una TV più intrattenitiva che educativa, perde la partita con le esigenze del nuovo marketing televisivo. Al suo posto arriveranno gli spot, snelli e veloci, che accompagneranno la società verso quella che Umberto Eco (1983) indicherà come neotelevisione, ovvero la TV commerciale degli Anni 80.

\section{Italianità in Carosello}

Lanalisi di Carosello attraverso il prisma dell'italianità è possibile se si considerano i complessi rapporti bilaterali esistenti fra storia, società, telespettatori e pubblicità stessa come due tipologie relazionali distinte ma interdipendenti: una è esterna, ovvero congiunge Carosello alla realtà; l'altra è interna e comprende i diversi livelli di soluzioni espressive utilizzate per la creazione del messaggio.

\subsection{Italianità esterna}

La situazione economico-storico-sociale, oltre a fare da contesto, entra in relazione con Carosello in una duplice modalità.

3 Dorfles (1998: 19). 
La collocazione di Carosello nel palinsesto televisivo rappresentò per molti bambini dell'epoca l'ultimo programma da vedere prima di andare a dormire, visto che dopo di esso iniziavano i programmi "per gli adulti". Da qui la celebre la frase: a nanna dopo Carosello! Non a caso i caroselli animati e la lunga serie di personaggi puntavano proprio ad affascinare i più piccoli che costituivano l'esca per poi arrivare a convincere i genitori all'acquisto. Nel primo decennio di trasmissione, agli occhi dello spettatore (anche adulto), Carosello non era soltanto pubblicità televisiva, ma "un mondo fiabesco dove regnavano la felicità e il benessere, un mondo dunque affascinante per una popolazione come quella italiana che proveniva da un lungo periodo di povertà e disagi" (Codeluppi 2013: 98-99), rappresentava uno specchio idilliaco che mostrava una società ideale, che tutti avrebbero voluto conquistare. Nel 1976, in un articolo per L'Espresso ${ }^{4}$, Eco sostiene come si trattasse di uno dei rari casi nella storia della società dei consumi in cui il pubblico, anziché subire la pubblicità, la desidera e la richiede. Era dunque un'italianità indotta, coadiuvata dalla dominante ripetitività tipica della comunicazione pubblicitaria, che ha plasmato e modernizzato gli italiani della fine degli Anni 50, aprendo gli occhi sulle altre realtà che li circondavano, mostrando al consumatore nuove esigenze, comodità e prodotti. Primi fra tutti i frigoriferi, grazie ai quali gli alimenti deperibili sono conservati più a lungo, limitando l'acquisto di cibi in scatola, anche in virtù dei nuovi grandi supermercati che consentono di fare la spesa non più ogni giorno, ma settimanalmente. Gli abitudinari lavori domestici vengono agevolati da lavatrici e macchine da cucire. A questi, ovviamente, si aggiunge l'infinita schiera di detersivi, additivi, ammorbidenti, prodotti per la casa nonché ogni tipo di utensile. Ci furono poi caroselli per ogni sorta di alimentari, medicinali e alcolici, fra i quali molti amari - come Ramazzotti, China Martini, Cynar (presente sin dal primissimo episodio), Zucca e Bergia - vermouth, whiskey, fino ad arrivare al vino che nel 1974 diverrà un prodotto sinonimo di italianità nella serie per la Chianti Ruffino dal titolo (e slogan) È un modo di fare cultura anche questo. I valori e i comportamenti-modello passano anche attraverso gli ambienti mostrati nei caroselli che sono sempre in ordine, puliti, moderni e funzionali, arredati con un certo gusto. Allo stesso modo - in maniera tipicamente pubblicitaria - le coppie e le famiglie che le abitano sono spesso felici, ben vestite, eleganti, sorridenti. Con il boom economico, i consumi di beni primari non devono più essere indotti dalla pubblicità, ma sono gli italiani stessi a cercare risposte ai propri nuovi bisogni. Si moltiplicano i prodotti per l'igiene e la cura del corpo, l'abbigliamento, i nuovi elettrodomestici, fino alle automobili e alla benzina.

4 “Qui telesera: Topogigio vi parla dell’aborto”, L’Espresso, 1ªgosto 1976. 
Quando, nella seconda metà degli Anni 60, nella società italiana iniziano la contestazione operaia e studentesca, nonché gli anni di piombo, Carosello, in una parte di serie prodotte, sembra farsi specchio riflesso della società italiana, probabilmente al fine di cercare di catturare ancora l'attenzione di quei giovani che 10 anni prima, da bambini, erano affascinati da quel mondo televisivo fatato, che voleva mostrarsi ai loro occhi ancora al passo coi tempi. Per questo molti Caroselli sfruttano nel pezzo canzoni e cantanti del momento o rappresentano scene studiate ad hoc con protagoniste le giovani generazioni dell'epocas.

Il ventennio caroselliano mostra inoltre l'emancipazione delle donne. Inizialmente rappresentate come massaie, fascia di consumatrici a cui Carosello si rivolge direttamente poiché perno dell'organizzazione domestica e dalle quali dipendeva l'acquisto di alimenti e prodotti per la casa, progressivamente le donne conquistano una maggiore libertà. Anche grazie al maggiore tempo libero guadagnato dal progresso tecnologico, abbandonano la vita casalinga e in Carosello le ritroviamo più sovente a lavorare e fare carriera in uffici, vestendo in maniera sempre meno castigata. La rivoluzione sessuale di quegli anni si tradurrà anche in pubblicità allusive, come quella nella quale la bionda modella Solvi Stübing abbraccia un'enorme bottiglia accompagnata dal claim Chiamami Peroni, sarò la tua birra!, che pare dare inizio alla quella deformazione dei valori che porterà lentamente all'uso pubblicitario della donna-oggetto (del desiderio).

\subsection{Italianità interna}

L'italianità di Carosello risiede innanzitutto nella sua forma unica, innovativa e 100\% made in Italy, riconosciuta nel 1972 negli Stati Uniti come la più specifica forma di pubblicità televisiva esistente (Calabrese 1975: 74-75). Al suo interno sono rintracciabili altri elementi caratterizzanti.

La sigla, innanzitutto, è accompagnata per tutto il ventennio caroselliano da una tarantella, forma musicale che riconduce alla napoletanità. Inoltre, la nuova versione del 1962 rende "più italiana" la sigla originaria sostituendo i sipari decorati a immagini di quattro famose piazze: Venezia, Siena, Napoli e Roma (Giusti 1995: 23-24). Dall'analisi dei singoli episodi si è notato come nel codino molto spesso ricorrano riferimenti diretti alle fabbriche dove vengono realizzati i prodotti pubblicizzati e alla loro provenienza: un modo per garantirne la qualità, puntando inoltre alla creazione di un rapporto di fiducia fra consumatore e marchio, contribuendo inoltre alla costituzione della sua identità di italiano.

5 Si veda ad esempio il carosello del 1970 della Cera Emulsio (www.youtube. com/watch?v=UwocgDUWwpw). 
In secondo luogo, fu fondamentale il duplice ruolo di personaggi animati ${ }^{6}$ e attori comici, teatrali, televisivi e cinematografici ${ }^{7}$ : se da un lato contribuivano a fidelizzare il consumatore, garantendo grazie alla loro notorietà la qualità del prodotto (creando inoltre i primi fenomeni televisivi di testimonial pubblicitari), dall'altro si sono fatti portatori del proprio bagaglio linguistico e culturale basato su modelli popolari, varietà dialettali o alte. Benché ognuno di essi avesse una frase o una serie di battute fisse che fungevano da aggancio ripetitivo e mnemonico al marchio ${ }^{8}$, accadeva che i personaggi monopolizzavano l'attenzione a tal punto che il prodotto (circoscritto agli ultimi secondi dei caroselli) raramente era memorizzato dal pubblico (Codeluppi 2013: 100).

\subsubsection{Italianità (socio)linguistica}

Lattenzione alla lingua italiana alla base del fine didattico della paleotelevisione è rintracciabile anche in Carosello sin dalle prime serie. Oltre ad un linguaggio più comune, con una prosa sciolta e più vicina al parlato, troviamo episodi nei quali le scelte stilistico-linguistiche propongono al telespettatore (poco istruito, nel primo decennio) modelli poetici, colti o in versi in stile retrò che ben si accompagnavano alle scenette teatrali. Per esempio, Giorgio Albertazzi recita brani famosi di commedie e poesie per la Barilla (come nell'episodio del 1959 dedicato a Tanto gentil e tanto onesta pare di Dante). Una delle serie di maggior successo, andata in onda dal 1958 al 1964, è Fierezza e nobiltà di Alemagna nella quale Lia Zoppelli e Enrico Viarisio interpretano due nobili

6 Fra i quali La Linea (Lagostina), Jo Condor, Jo Galassia, Olivella e Mariarosa (olio Bertolli), l’Omino coi Baffi (caffettiere Bialetti), il Gigante amico (Ferrero), Calimero e l'Olandesina (detersivo Miralanza), Toto e Tata (Motta), Topo Gigio (Pavesini), nonché l'ippopotamo Pippo (pannolini Lines), Carmencita e il Caballero (caffè Paulista) e Papalla (elettrodomestici Philco) creati da Armando Testa e Armando Guidone.

7 Fra questi: Totò e Alberto Sordi, Mike Bongiorno, Carla Fracci, Raffella Carrà, Gino Bramieri, Dario Fo, Mina, Cesare Polacco, Renato Rascel, Ugo Tognazzi, Raimondo Vianello e Sandra Mondaini, Ernesto Calindri, Gino Cervi, Edoardo De Filippo, Aldo Fabrizi, Vittorio Gassman, Alberto Lionello, Amedeo Nazzari, Paolo Panelli.

8 Ad esempio, l'ispettore Rock che al commento "Lei è un fenomeno, ispettore. Non sbaglia mai!", si toglieva con il cappello mostrando la sua calvizie dicendo: "Non è esatto! Anch'io ho commesso un errore, non ho mai usato la brillantina Linetti". O l'intera struttura ripetitiva del carosello del caffè Paulista: dall'introduttivo "Nella pampa sconfinata, dove le pistole dettano legge..." alla ricorrente domanda del Caballero "Carmencita abita qui?" e, una volta trovata, all'invito "Bambina, sei già mia! / Chiudi il gas e vieni via!”, cui la ragazza rispondeva inevitabilmente "Pazzo! L'uomo che amo è un uomo molto in vista. / È forte, è bruno e ha il baffo che conquista!" 
che, parlando in orecchiabili e mnemonici ottonari, sistematicamente finiscono per litigare. Il pezzo però, come nel frammento tratto dall'episodio Il fotografo del 1957, termina sempre con l'immancabile lieto fine con una struttura che rimarrà pressoché costante per l'intera serie (in neretto):

LEI: Lei, Barone Vladinotte, / è uno stolto Don Chisciotte!

LUI: Son parole assai severe. / Lei bistratta un cavaliere / con durezza e incomprensione.

LEI: Via, non pianga, bamboccione.

LUI: Sono offeso e umiliato.

LEI: Su, le offro un buon gelato.

LUI: Ma è un gelato di Alemagna! / Ullallà è una cuccagna!

LEI: I suoi gusti son squisiti!

IL GELATAIO: I signori son serviti!

Il confetto lassativo Falqui ha fondato sulla parola la sua intera serie. A parte il celeberrimo e azzeccatissimo slogan creato da Marcello Marchesi Basta la parola, ogni carosello girava intorno alla ricerca del significato corretto di lemmi meno comuni (fra cui melomane, torrido, piraña, trifora, mesone, spasimante) terminando immancabilmente con il sillogismo se non è stato facile trovare il significato giusto di questa parola, in altri casi sarà più facile, come con il confetto Falqui, perché: basta la parola. Oltre ad essere un ottimo esempio di retorica persuasiva, il carosello della Falqui può aver contribuito in un certo modo alla coscienza linguistica degli italiani dell'epoca.

Sulla scia del successo delle commedie all'italiana e del teatro dialettale (ritrasmesso anche in televisione) non di rado in Carosello personaggi reali e animati ricorrono a forme dialettali attenuate comprensibili. Da un lato attori e comici - fra i quali Gilberto Govi (genovese), Macario (torinese), i De Filippo (napoletano), Gino Bramieri (milanese), Aldo Fabrizi (romanesco) - portano sulla scena il proprio dialetto accompagnato dalle caratteristiche stereotipate tipiche. In altri casi il dialetto è presente per tracciare il carattere di un certo personaggio, come quando per la Moplen Gino Bramieri interpreta un veneziano stereotipicamente amante dei vini e dall'ubriacatura facile: si notano la caduta delle consonanti in posizione intervocalica e forme morfosintattiche connotate (Quando bevo l'agua, vuol di' proprio che go perso 'a testa!). L'italiano regionale o più dialettale della gente comune compare, per esempio, nel "neorealismo" del carosello per la Dash nel quale Paolo Ferrari, con la scusa di dover recitare una poesia in dialetto, chiede ai passanti di leggere e tradurre il testo, così da dimostrare che ad un attore famoso non si rifiuta mai niente... a parte lo scambio di un fustino di detersivo Dash per due di uno comune. Le varietà dialettali possono inoltre essere lo spunto per creare un tormentone, come le battute 
in milanese e il jingle della celebre serie per China Martini Düra minga, düra no con Ernesto Calindri e Franco Volpi: si noti la tipica vocale turbata [ü] e il rafforzativo della negazione minga. Ritroviamo lo stesso Volpi con Giovanna Ralli nella serie Nannina a Milano per la Locatelli del 1960 nella quale l'uso di elementi dialettali veicola il confronto linguistico e sociale fra coniugi. Più numerosi i tratti del romanesco di Nannina. A livello fonetico si riportano: la geminazione delle consonanti (cossi), la realizzazione del gruppo <gn> come [n:] al posto di [ndz] o [nj] (gnente) e la spirantizzazione di [t $]$ in [ $\left.\int\right]$ in posizione intervocalica (velo[S]e). A livello morfosintattico: apocope degli infiniti e degli appellativi (parla', a Nanni'), uso di forme verbali connotate (dimo $>$ diciamo, aspettame $>$ aspettami, annamo $>$ andiamo, annate $>$ andate), varie parti del discorso nella forma romanesca (pronomi e preposizioni in $-e$, aferesi dell'articolo indeterminativo) o che con valore interrogativo. A livello lessicale si trova indove. Il milanese del marito è perlopiù marcato foneticamente dalla realizzazione aperta della vocale [e] in [per'ke] e dal troncamento della vocale finale, mentre nelle frasi te vedet e la vorêva insegnâm l'italiano! spiccano forme morfosintattiche connotate (il pronome soggetto $l a$, quello di termine enciclico $-m$ ).

Dialettalità accompagnate dal bagaglio di luoghi comuni consolidati nell'immaginario degli italiani appaiono anche nel linguaggio dei personaggi animati. Nel jingle della serie per i biscotti Talmone Miguel e il coro usano tratti dei dialetti veneti, in particolare forme tipiche verbali dell'indicativo presente e l'apocope della vocale atona finale (l'è lì, l'è là, l'è là che l'aspettava, l'è li che aspettava Miguel... Miguel son mi! e ti, e ti, e ti no dixe niente, e ti dixe niente a Miguel...?). Il romanesco - marcato qui da rotacismo - torna con Riccardone, protagonista della serie per l'insetticida Super Faust (Io sono er bullo atomico / me chiamo Riccardon / sono un bullo d'eccezion! / E mica son fasullo / Sono un vero superbullo! / E se m'arabbio divento un lupo! / Ma chi te vole? Ma chi te conosce?) e con Gregorio er guardiano der pretorio (tecnofibre Rhodiatoce). I pirati dei caroselli dell'Amarena Fabbri sono marcati da tratti tipici di tre varietà: il torinese di Salomone Pirata Pacioccone (Porta pasiensa!), il siciliano di Mano di Fata (Capitano, il qui presente si rrrrifiuta di collaborare. / Lo possiamo torturare? / L'elisir di lunga vita teniamo! / A tennis sta ggiocando) e il veneziano di Fortunato Pirata Scalognato (Poreto! / Orco can! L'a xe meso un abito pesante! / Ostregheta! / La corda de sicuresa!). In altri casi si tratta di tracce prosodiche di genovese (Capitan Trinchetto per la Recoaro), di toscano (Zaccaria diavolo di terza categoria per la Minerva), di siciliano (il vigile del Brodo Lombardi che ripete "Che fa? Concilia?"), di veneziano e di padovano (rispettivamente di Calimero e della mamma che, nomen omen, è una gallina padovana; detersivo Ava). 


\subsubsection{Scelte linguistico-retoriche: slogan, neologismi, frasi idiomatiche}

Il discorso pubblicitario, in quanto persuasivo, è unargomentazione retorica. Non stupisce che la struttura della pubblicità e di Carosello sembri ricalcare la struttura organizzativa aristoteliana. Esordio, narratio, confirmatio ed epilogo corrispondono a presentazione del prodotto, pezzo, conclusione e dimostrazione delle prove (frase d'aggancio), codino e slogan (Calabrese 1975: 126-128).

Se da un lato l'italianità di Carosello è evidente nel grande gioco creativo messo in atto nel codice verbale, visivo e musicale, anche in virtù della ripetitiva serialità, la retorizzazione di testi e slogan consente la memorizzazione, permettendone l'introduzione nell'uso comune, come è accaduto per esempio con Contro il logorio della vita moderna e Basta la parola.

Numerosi sono gli strumenti retorici utilizzati in Carosello. Un'analisi di ogni fenomeno necessiterebbe di uno spazio non idoneo a questa sede. Oltre a quelli già citati, riportieremo dunque alcune esemplificazioni fra le più ricorrenti. Figure molto frequenti sono: la rima, spesso con il marchio ( $S$, signora guardi ben / che sian fatti di Moplen; Ava, come lava!; Ma è un prodotto di Alemagna! / Ullallà è una cuccagna!), l'allitterazione (Arriva, arriva Rivarossi), l'anafora (Pavesini: sani, leggeri, nutrienti. Pavesini: dateli con fiducia ai vostri bimbi. Pavesini: la prima dolcezza della vita). Soventi sono le soluzioni basate su anfibologie (Non è vero che tutto fa brodo, brodo Lombardi; Con quella bocca può dire ciò che vuole, dentifricio Chlorodont), neologismi (Simmenthal... mente buona!; Ramazzottimista) e prosopopee (Chiamami Peroni, sarò la tua birra). L'identità italiana è consolidata anche attraverso enunciati costruiti sull'antitesi io-gli altri stimolando lo spettatore a imitare comportamenti trasmessi dal messaggio promozionale come socialmente favorevoli per chi deciderà di acquistare quel prodotto (Ma che figura! Chi se ne intende chiede Stock!).

\section{Conclusioni}

Lo studio condotto su una selezione di caroselli risalenti all'intero ventennio ha permesso di individuare una serie di tratti riconducibili a particolari modalità relazionali di espressione e riflesso dell'italianità. È evidente la polifunzionalità di Carosello: per tutti gli italiani era un appuntamento quotidiano irrinunciabile (funzione fàtica) che ha mutato comportamenti e abitudini (funzione conativa), aiutandoli ad uscire dalla desolazione della guerra fino a condurli al boom economico (Caniglia 2017: 9-10).

Sembra dunque che in Carosello la retorica persuasione tipica del mezzo pubblicitario si sia sovrapposta ad una reiterazione non solo dei comunicati promozionali, bensì di un sistema "moralistico-pedagogico" (Calabrese 1975: 183) di nuovi valori, modelli e comportamenti positivi da seguire che hanno 
guidato gli italiani verso la maturità della società dei consumi. Successivamente, una volta adempiuto a questo compito educativo e a causa delle criticità che ne determinarono la chiusura, Carosello si è fatto specchio riflesso della società italiana, limitando la propria funzione a quella di intrattenimento. Ciononostante, ha lasciato in eredità agli italiani un grande patrimonio culturale e linguistico radicato nella memoria comune, non solo delle generazioni che andavano a letto dopo Carosello.

\section{Bibliografia}

Alessandri, Alessandra (1994). "L'eredità di Carosello", Campagne, XI, pp. 92-95.

Calabrese, Omar (1975). Carosello o dell'educazione serale, [in] Cimorelli-Roffi (a c. di), Firenze, Cooperativa Libraria Universitatis Studii Florentini, pp. 55-189.

Calabrese, Stefano (2008). Retorica del linguaggio pubblicitario, Bologna, Archetipolibri.

Caniglia, Federica (2017). "Pubblicità televisiva: Carosello, 'La favola del consumo", Filosofia e nuovi sentieri, 22 gennaio 2017. Testo disponibile allindirizzo: https:// filosofiaenuovisentieri.com/2017/01/22/pubblicita-televisiva-carosello-la-favola-delconsumo/ [27/06/2019].

Cimorelli, Dario e Roffi, Stefano (a c. di) (2019). Carosello. Pubblicità e televisione 1957-1977, Milano, Silvana Editoriale.

Codeluppi, Vanni (2013). Storia della pubblicità in Italia, Roma, Carocci.

De Mauro, Tullio (1993). Storia linguistica dell'Italia unita, Roma-Bari, Laterza (1a ed. 1963).

Dorfles, Piero (1998). Carosello, Bologna, il Mulino.

Eco, Umberto (1983). "Tv: la trasparenza perduta", [in] U. Eco, Sette anni di desiderio, Milano, Bompiani, pp. 163-179.

Giusti, Marco (1995). Il grande libro di Carosello, Piacenza, Sperling \& Kupfer.

Magagnoli, Stefano (2013). "Carosello, ovvero l'educazione al consumo", Parma economica, 3, pp. 32-38.

Melegaro, Marco (2017). Carosello. Genio e pubblicità all'italiana, Milano, Novecento.

Molinari, Vito (2019). Carosello... e poi tutti a nanna. 1957-1977: i vent'anni che hanno cambiato l'Italia, Sestri Levante, Gammarò Edizioni.

Pittèri, Daniele (2015). “Sotto il segno di 'Carosello', 1954-1979”, [in] La pubblicità in Italia. Dal dopoguerra a oggi, Roma-Bari, Laterza.

Simone, Raffaele (1987). "Specchio delle mie lingue", Italiano e oltre, 2, pp. 53-59.

Simonelli, Giorgio e Colombo, Elena (2004). "1954-2004: Fenomenologia di mezzo secolo di televisione", Ambiente, Società, Territorio, 01/2004. Testo disponibile all'indirizzo: aiig.it/ OLD_giugno2015/documenti/rivista/2004/n1/n1_13.pdf [27/06/2019]. 